\title{
THE $q^{2}$ DEPENDENCE OF $W$ AND $Z$ COUPLING CONSTANTS IN THE INTERVAL $0 \leq\left|q^{2}\right| \leq m_{Z}^{2}$
}

\author{
V.A. Novikov*) and L.B. Okun*) \\ Theoretical Physics Division, CERN \\ CH-1211 Geneva 23 \\ and \\ M.I. Vysotsky \\ ITEP, 117259 Moscow, Russia
}

\begin{abstract}
The gauge coupling constants of $W$ and $Z$ bosons, $\alpha_{W}$ and $\alpha_{Z}$, are not running unless $\left|q^{2}\right| \gg m_{Z}^{2}$. Their dependence on $q^{2}$ is given by simple expressions and is very weak in the interval $0 \leq q^{2} \leq m_{Z}^{2}$ compared to that of the photon.
\end{abstract}

PQDQอ1237

*) Permanent address: ITEP, 117259 Moscow, Russia.

CERN-TH.7153

January 1994 
The phenomenon of "running" (logarithmic dependence on the momentum transfer $q^{2}$ ) has been known for about four decades [1], [2] as regards $\alpha\left(q^{2}\right)$, the coupling constant of photons to charged particles. In a number of papers this property was also attributed to the gauge couplings of $W$ and $Z$ bosons, $\alpha_{W}\left(q^{2}\right)$ and $\alpha_{Z}\left(q^{2}\right)$. Even a running Fermi coupling constant $G_{F}\left(q^{2}\right)$ has been discussed in the literature.

It was stressed in Ref. [3] that $\alpha_{W}\left(q^{2}\right)$ and $\alpha_{Z}\left(q^{2}\right)$, contrary to $\alpha\left(q^{2}\right)$, do not run, but "crawl", and that they are therefore, up to corrections of the order of $\alpha$, the same at $q^{2} \simeq m_{Z}^{2}$ and at $q^{2} \simeq 0$. As was also stressed in Ref. [3] the $\nu_{\mu}$ e scattering amplitude does not contain logarithmically large contributions to the neutrino electromagnetic radius: these contributions practically cancel each other. Hence one can extract the coupling of the $Z$ boson to the muon neutrino, $g^{\nu_{\mu}}$, by combining CHARM II data on the $\nu_{\mu} e$ scattering amplitudes $g_{A . V}^{e \nu_{\mu}}(0)$ and the LEP data on the coupling of the $Z$ boson to the clectron, $g_{A, V}^{e}$.

Indeed if we neglect non-logarithmic corrections of the order of $\alpha / \pi$ the amplitudes for $Z$ decay can be written in the form

$$
\begin{array}{r}
T\left(Z \rightarrow \nu_{\mu} \tilde{\nu_{\mu}}\right)=\frac{1}{2} f^{\nu_{\mu}} Z_{\alpha} \bar{\nu} \gamma_{\alpha}\left(1+\gamma_{5}\right) \nu, \\
T(Z \rightarrow e \tilde{e})=\frac{1}{2} Z_{\alpha} \bar{e} \gamma_{\alpha}\left(f_{V}^{e}+f_{A}^{e} \gamma_{5}\right) e
\end{array}
$$

where $f^{\nu_{\mu}}=f g^{\nu_{\mu}}, f_{A, V}^{e}=f g_{A, V}^{e}$, and axial and vector couplings $g_{A, V}$ are equal in this approximation to

$$
g_{A}=T_{3}, g_{V}=T_{3}-2 Q s^{2}
$$

where $T_{3}$ is the third projection of the isospin and $s^{2}=0.2312$ [4]. In all our previous papers the $Z$-boson coupling constant $f$ was defined at $q^{2}=m_{Z}^{2}$ by the equation:

$$
f^{2}=4 \sqrt{2} G_{\mu} m_{Z}^{2}=0.54864(8) .
$$

In this paper we will consider $f\left(q^{2}\right)$ and will use eq. (2) as the definition of $f\left(m_{Z}^{2}\right)$. We will denote by $e Q$ the coupling of the photon to the charge $Q$, and the coupling of the $W$ boson to the charged current by $g / \sqrt{2}$. It is convenient to consider: $\alpha\left(q^{2}\right)=e^{2}\left(q^{2}\right) / 4 \pi, \alpha_{W}\left(q^{2}\right)=g^{2}\left(q^{2}\right) / 4 \pi, \alpha_{Z}\left(q^{2}\right)=f^{2}\left(q^{2}\right) / 4 \pi$.

The statement that $\alpha_{Z}\left(q^{2}\right)$ does not run in the region $\left|q^{2}\right| \leq m_{Z}^{2}$ means that

$$
\alpha_{Z}(0) \simeq \alpha_{Z}\left(m_{Z}^{2}\right)
$$

The $\nu_{\mu} e$ scattering is described by phenomenological amplitudes $g_{V, A}^{e \nu_{\mu}}\left(q^{2}\right)$.

$$
T_{e \nu_{\mu}}=\frac{G}{\sqrt{2}} \bar{\nu} \gamma_{\alpha}\left(1+\gamma_{5}\right) \nu \bar{e}\left[g_{V}^{e \nu_{\mu}} \gamma_{\alpha}+g_{A}^{e \nu_{\mu}} \gamma_{\alpha} \gamma_{5}\right] e
$$


If we neglect small non-logarithmic one-loop corrections and take into account only those corrections that potentially could push $\alpha_{Z}\left(q^{2}\right)$ to run, we get for $T_{e \nu_{\mu}}$

$$
\begin{aligned}
T_{e \nu_{\mu}} & =\frac{1}{4} f^{2}\left(q^{2}\right) \frac{1}{q^{2}-m_{Z}^{2}} \bar{\nu} \gamma_{\alpha}\left(1+\gamma_{5}\right) \nu \times \\
& \times \quad \bar{e}\left[g_{V}^{e} g^{\nu_{\mu}} \gamma_{\alpha}+g_{A}^{e} g^{\nu \mu} \gamma_{\alpha} \gamma_{5}\right] e .
\end{aligned}
$$

If there is no substantial $\alpha_{Z}\left(q^{2}\right)$ evolution from $q^{2}=0$ to $q^{2}=m_{Z}^{2}$ (see eq. (3)) we get

$$
g_{V, A}^{e \nu_{\mu}}(0) \simeq 2\left[\frac{f^{\nu_{\mu}}\left(m_{Z}^{2}\right)}{f\left(m_{Z}^{2}\right)}\right]\left[\frac{f_{V, A}^{e}\left(m_{Z}^{2}\right)}{f\left(m_{Z}^{2}\right)}\right]=2 g^{\nu_{\mu}} g_{V, A}^{e} .
$$

By using their own data and, in addition, LAMPF data on $\nu_{e} e$ scattering and the total invisible width of the $Z$ boson, the CHARM II collaboration succeeded [5] in determining the couplings of $Z$ to all three types of neutrinos:

$$
\begin{aligned}
& 2 g^{\nu_{\mu}}=1.00(3), \\
& 2 g^{\nu_{e}}=1.02(14), \\
& 2 g^{\nu_{r}}=0.98(15) .
\end{aligned}
$$

In view of the importance of this test of the universality of the neutrino couplings, it seems necessary to derive explicit equations describing the dependence of $\alpha_{Z}$ and $\alpha_{W}$ on $q^{2}$ in order to estimate the accuracy of equation (6) and of similar equations for $g_{A, V}^{e \nu_{\mu}}$ on which the results (7)-(9) are based [5].

In what follows we will neglect masses of fermions by considering $q^{2} \gg$ $m_{f}^{2}$. We will also omit the $W$ and $Z$ boson loop contributions to $\alpha_{Z}\left(q^{2}\right)$ and $\alpha_{W}\left(q^{2}\right)$, because they are not logarithmically enhanced for $\left|q^{2}\right| \leq m_{Z}$.

As usual, we will start with a bare gauge coupling $g_{0}$ and bare boson mass $m_{0}$ and by summing up a geometric progression with self-energy insertions $\sum\left(q^{2}\right)$ will obtain the $q^{2}$-dependent physical gauge coupling:

$$
g^{2}\left(q^{2}\right)=\frac{g_{0}^{2}}{1+g_{0}^{2} \frac{\left.\sum\left(q^{2}\right)-\sum\left(m^{2}\right)\right)}{\left(q^{2}-m^{2}\right)}},
$$

where $m$ is the physical mass. It is possible to get rid of $g_{0}$ by subtraction, e.g. $1 / g^{2}\left(q^{2}\right)-1 / g^{2}(0)$. Hence:

$$
\frac{1}{\alpha_{Z}\left(q^{2}\right)}-\frac{1}{\alpha_{Z}(0)}=b_{Z} F(x), \text { where } x=\frac{q^{2}}{m_{Z}^{2}},
$$




$$
\begin{gathered}
\frac{1}{\alpha_{W}\left(q^{2}\right)}-\frac{1}{\alpha_{W}(0)}=b_{W} F(y), \text { where } y=\frac{q^{2}}{m_{W}^{2}}, \\
F(x)=\frac{x}{1-x} \ln |x| .
\end{gathered}
$$

One can easily check that

$$
F(-1)=F(0)=0, \quad F(+1)=-1,
$$

and

$$
F((-0.2785)=+0.2785
$$

is the maximal value of $F(x)$. As for the coefficients $b_{Z}$ and $b_{W}$, they contain contributions from quarks and leptons:

$$
\begin{aligned}
b_{Z} & =\frac{1}{48 \pi}\left\{2 \cdot 3\left[1+\left(1-8 s^{2} / 3\right)^{2}\right]+\right. \\
& \left.+3 \cdot 3\left[1+\left(-1+4 s^{2} / 3\right)^{2}\right]+3\left[1+\left(1-4 s^{2}\right)^{2}\right]+3 \cdot 2\right\}= \\
& =\frac{1}{16 \pi}\{2 \cdot 1.147+3 \cdot 1.149+1.006+2\}=0.195,
\end{aligned}
$$

where the first term is the up-quarks contribution, the second the downquarks one, the third one comes from the charged leptons, while the fourth comes from neutrinos:

$$
b_{W}=\frac{1}{24 \pi}\{2 \cdot 3 \cdot 2+3 \cdot 2\}=\frac{3}{4 \pi}=0.239,
$$

where the first term is the quark and the second is the lepton contribution.

Let us now remind [4] that

$$
\begin{gathered}
\alpha_{Z}\left(m_{Z}^{2}\right)=\alpha\left(m_{Z}^{2}\right) / c^{2} s^{2}=\frac{\sqrt{2} G_{\mu} m_{Z}^{2}}{\pi}=1 / 22.905(4) \\
\alpha_{W}\left(m_{Z}^{2}\right)=\alpha\left(m_{Z}^{2}\right) / s^{2}=1 / 28.74 \\
\alpha\left(m_{Z}^{2}\right)=1 / 128.87(10) \\
c^{2} s^{2}=\frac{\pi \alpha\left(m_{Z}^{2}\right)}{\sqrt{2} G_{\mu} m_{Z}^{2}}=0.17774(2) \\
s^{2}=0.2312(3), c^{2}=0.7688(3)
\end{gathered}
$$

Thus

$$
\begin{gathered}
1 / \alpha_{Z}\left(m_{Z}^{2}\right)-1 / \alpha_{Z}(0)=-0.195, \\
1 / \alpha_{Z}(0)=22.905+0.195=23.1,
\end{gathered}
$$




$$
\begin{gathered}
1 / \alpha_{W}\left(m_{Z}^{2}\right)-1 / \alpha_{W}(0)=0.239 F\left(\frac{1}{c^{2}}\right)= \\
=0.239 F(1.3)=-0.239 \cdot 1.137=-0.272, \\
1 / \alpha_{W}(0)=28.74+0.272=29.01 .
\end{gathered}
$$

One can see that from $q^{2}=0$ to $q^{2}=m_{Z}^{2} \alpha_{Z}$ increases by $0.85 \%$, while $\alpha_{W}$ by $0.95 \%$. This should be compared with the running of the photon coupling [1]:

$$
\frac{1}{\alpha\left(m_{Z}^{2}\right)}-\frac{1}{\alpha(0)}=128.87-137.04=-8.17
$$

which represents an increase by $6.34 \%$. For large $q^{2}\left(q^{2} \gg m_{f}^{2}\right)$ the $q^{2}$ dependence of $1 / \alpha\left(q^{2}\right)$ is approximately logarithmic:

$$
\frac{1}{\alpha\left(q^{2}\right)}-\frac{1}{\alpha(0)} \simeq-8.17+\frac{20}{9 \pi} \ln \frac{m_{Z}^{2}}{q^{2}} .
$$

Here

$$
\frac{20}{9 \pi}=\frac{1}{3 \pi} \sum_{f} Q_{f}^{2}=\frac{1}{3 \pi}\left[3+\frac{4}{9} \cdot 3 \cdot 2+\frac{1}{9} \cdot 3 \cdot 3\right] .
$$

Neglecting fermion masses, especially $m_{b}, m_{r}, m_{c}$, leads to an artifact: the right-hand side of eq. (28) vanishes at $q^{2}=(0.28 \mathrm{GeV})^{2}$, not at $q^{2}=0$.

From the $1 \%$ difference between $\alpha_{Z}(0)$ and $\alpha_{Z}\left(m_{Z}^{2}\right)$ one would naïvely expect that coefficient 2 in Eq. (6) should be multiplied by 0.99 , which would change 1.00 in eq. (3) to 1.01. However, other radiative corrections may compensate this $1 \%$ change.

\section{Acknowledgments}

We thank $k$. Winter for showing us the preliminary version of ref. [5]. Two of us (V.N. and L.O.) thank the CERN Theory Division for their warm hospitality. We are grateful to the Russian Foundation for Fundamental Research for grant 93-02-14431. We are grateful to V.L. Telegdi who insisted on presenting the behaviour of $\alpha_{Z}$ and $\alpha_{W}$ not only in $q$-space, but also in $x$-space. Unfortunately we did not succeed in obtaining a graphic picture in $x$-space. 


\section{References}

[1] L.D.Landau, A.A.Abrikosov, and I.M.Khalatnikov. Dokl. Akad. Nauk SSSR $\underline{95}$ (1954) 497, 773, 1177.

[2] M.Gell-Mann, F.Low. Phys.Rev. 95 (1954) 1300.

[3] V.A.Novikov, L.B.Okun, and M.I.Vysotsky. Phys.Lett. B298 (1993) 453.

[4] V.A. Novikov, L.B.Okun, and M.I.Vysotsky. Modern Phys.Lett. A $\underline{8}$ (1993) 2529, E.3301.

[5] The CHARM II Collaboration, P.Vilain et al. Preprint CERN-PPE/93186, submitted to Physics Letters B. 